\title{
Imaging of persistent CAMP signaling by internalized G protein-coupled receptors
}

\author{
Davide Calebiro, Viacheslav O Nikolaev and Martin J Lohse \\ Rudolf Virchow Center, DFG-Research Center for Experimental Biomedicine and Institute of Pharmacology and Toxicology, University of Würzburg, Versbacher Straße 9, \\ 97078 Würzburg, Germany \\ (Correspondence should be addressed to D Calebiro; Email: davide.calebiro@ toxi.uni-wuerzburg.de)
}

\begin{abstract}
G protein-coupled receptors (GPCRs) are the largest family of plasma membrane receptors. They mediate the effects of several endogenous cues and serve as important pharmacological targets. Although many biochemical events involved in GPCR signaling have been characterized in great detail, little is known about their spatiotemporal dynamics in living cells. The recent advent of optical methods based on fluorescent resonance energy transfer allows, for the first time, to directly monitor GPCR signaling in living cells. Utilizing these methods, it has been recently possible to show that the receptors for two protein/peptide hormones, the TSH and the parathyroid hormone, continue signaling to cAMP after their internalization into endosomes. This type of intracellular signaling is persistent and apparently triggers specific cellular outcomes. Here, we review these recent data and explain the optical methods used for such studies. Based on these findings, we propose a revision of the current model of the GPCR-CAMP signaling pathway to accommodate receptor signaling at endosomes.
\end{abstract}

Journal of Molecular Endocrinology (2010) 45, 1-8

\section{Introduction}

G protein-coupled receptors (GPCRs) constitute the major family of cell surface receptors. They comprise receptors for light, taste, and smell as well as receptors for ions, small transmitters, peptides, and large protein hormones. Given their involvement in fundamental biological processes and their accessibility, GPCRs serve as targets for major classes of drugs, including beta-blockers, antihistamines, and opiates (Pierce $e t$ al. 2002, Lagerstrom \& Schioth 2008).

Research done mostly in the 1980s using cells, cell membrane preparations, and reconstituted systems with purified proteins has revealed the basic structure and functional properties of GPCR signaling cascades. They consist of a receptor, a heterotrimeric $\mathrm{G}$ protein with $\alpha$-, $\beta$-, and $\gamma$-subunits, and an effector (Pierce $e t$ al. 2002). The effector can be an enzyme, such as adenylyl cyclase, which produces the soluble second messenger cAMP, or an ion channel, such as the G proteinregulated inward rectifying $\mathrm{K}^{+}$-channel (Gilman 1987, Pierce et al. 2002). Upon prolonged stimulation, many GPCRs become phosphorylated by GPCR kinases (GRKs) and then recruit $\beta$-arrestins, events that are responsible for fast, so-called homologous signal desensitization (Lohse 1993, Pierce et al. 2002).
Subsequently, $\beta$-arrestins promote the internalization of receptors into endosomes (Lohse 1993, Pierce et al. 2002, Hanyaloglu \& von Zastrow 2008, Sorkin \& von Zastrow 2009). From there, they can become dephosphorylated and recycle to the cell surface so that signaling is restored (Lohse 1993, Yu et al. 1993, Pippig et al. 1995, Krueger et al. 1997). Alternatively, the receptors may be targeted for degradation, and the consequent reduction in receptor number, known as receptor downregulation, contributes to later phases of signal desensitization (Lohse 1993, Tsao et al. 2001). In addition, receptor internalization may also trigger 'nonclassical' signaling pathways, such as the mitogenactivated protein kinase (MAPK) cascade, at endosomes (Pierce et al. 2002, Slessareva et al. 2006, Diaz Anel 2007, Garcia-Regalado et al. 2008, Sorkin \& von Zastrow 2009). In contrast, 'classical' G protein-dependent cascades, such as those involving cAMP, are generally assumed to be activated exclusively at the cell surface.

Although many molecular steps of GPCR signaling have been characterized in great detail, little is known about their spatiotemporal dynamics in living cells. This is because biochemical techniques have limited temporal and, generally, no spatial resolution. To tackle these limitations, we and others have developed optical methods for visualizing various steps of GPCR-cAMP 
signaling directly in living cells (Lohse et al. 2008). These techniques have been instrumental for the recent finding that some GPCRs, such as the TSH and the parathyroid hormone (PTH) receptors, continue signaling to cAMP after internalization (Calebiro $e t$ al. 2009, Ferrandon et al. 2009). Here, we will review these recent results, highlighting the new possibilities offered by such optical methods. On the basis of these results, we propose a new model of GPCR signaling that takes into account signaling to cAMP by internalized receptors.

\section{Techniques for monitoring GPCR-CAMP signaling in living cells}

In the past few years, we and others have generated a series of genetically encoded fluorescent sensors that report upon key steps of GPCR signaling in living cells, ranging from ligand binding and receptor activation to the downstream generation of cAMP (Zaccolo et al. 2000, Janetopoulos et al. 2001, Bünemann et al. 2003, Vilardaga et al. 2003, DiPilato et al. 2004, Nikolaev et al. 2004, 2006, Ponsioen et al. 2004, Castro et al. 2005, Lohse $e t$ al. 2008). These sensors are largely based on the physical phenomenon of fluorescence resonance energy transfer (FRET), which can be measured by different microscopy techniques. Once introduced by transfection or other methods, these sensors allow the direct visualization of biochemical events in living cells. Since the temporal and spatial resolutions are those of conventional fluorescence microscopy, i.e. up to a few milliseconds in time and a few hundred nanometers in space, these techniques can capture fast signaling events and, at the same time, define their subcellular location.

FRET indicates the transfer of energy from one fluorophore (donor) to another (acceptor), and it takes place exclusively if the two fluorophores are in close proximity. Thus, it can be exploited to monitor interactions between two proteins or conformational changes within a single protein, provided that such proteins are labeled with a suitable pair of donor and acceptor fluorophores. Both types of FRET, i.e. interand intramolecular, have been employed for the generation of fluorescent sensors (see below).

A large and heterogeneous group of GPCRs utilizes cAMP as second messenger. Given the pivotal role of cAMP in GPCR signaling and its implication in several important biological processes, researchers have long attempted to generate sensors for this molecule. The first FRET reporter for cAMP (FlCRhR) was developed almost 20 years ago by Adams et al. (1991). This sensor was based on the dissociation of the catalytic $(C)$ from the regulatory (R) subunits of protein kinase A (PKA) upon binding of cAMP. By chemically labeling purified
$\mathrm{C}$ and $\mathrm{R}$ subunits with two different fluorophores and microinjecting them into cells, it was possible to follow the changes in the intracellular concentration of cAMP by monitoring FRET levels. Later, this sensor was modified by Zaccolo et al. (2000) to be genetically encoded by substituting the chemical fluorophores with two suitable color variants of the Aequorea victoria green fluorescent protein (GFP). This represented a major advantage, as the new sensor can be transfected into cells, without the need for complex purification, labeling, and microinjection procedures. Later on, single-chain sensors were described that are based on either the entire coding sequence of the exchange protein activated by cAMP (Epac; DiPilato et al. 2004, Ponsioen et al. 2004) or on just a single cAMP-binding domain derived from such cAMP-binding proteins (Nikolaev et al. 2004, 2006). The Epac1-camps sensor (Fig. 1A) is a typical example of the latter approach, which has the advantage of avoiding overexpression of an active signaling molecule such as PKA or Epac (Nikolaev et al. 2004, 2006). In this type of sensor, the cAMP-binding domain is flanked on each side by either of two popular GFP color variants, i.e. the cyan fluorescent protein (CFP) and the yellow fluorescent protein (YFP). Binding of cAMP to the sensor produces a conformational change that causes a reduction of FRET between CFP and YFP. Thus, FRET values are inversely related to the intracellular concentration of cAMP.

To measure cAMP levels in living native cells and tissues, we have recently generated transgenic mice with either tissue-specific (Nikolaev et al. 2006) or ubiquitous (Calebiro et al. 2009) expression of such cAMP sensors. The latter mice contain high levels of the Epac1-camps sensor in most tissues (Fig. 1B) and can be used to monitor GPCR-cAMP signaling in different types of primary cells (Calebiro et al. 2009).

\section{Direct monitoring of TSH signaling in intact thyroid follicles}

The thyroid gland is an interesting model for studying the spatiotemporal dynamics of GPCR-cAMP signaling. The anatomical and functional unit of thyroid tissue is the thyroid follicle, consisting of a monolayer of epithelial cells that defines an inner cavity, where thyroid hormones are synthesized and stored in the form of iodinated thyroglobulin (Fig. 2). Thyroid epithelial cells are under the strict control of TSH, secreted by the anterior pituitary (Magner 1990). TSH binds to a GPCR, the TSH receptor (TSHR), located on the basolateral membrane of thyroid epithelial cells (Vassart \& Dumont 1992). At physiological TSH concentrations, the TSHR is predominantly coupled to $G_{s}$, and TSH effects are largely mediated by the 

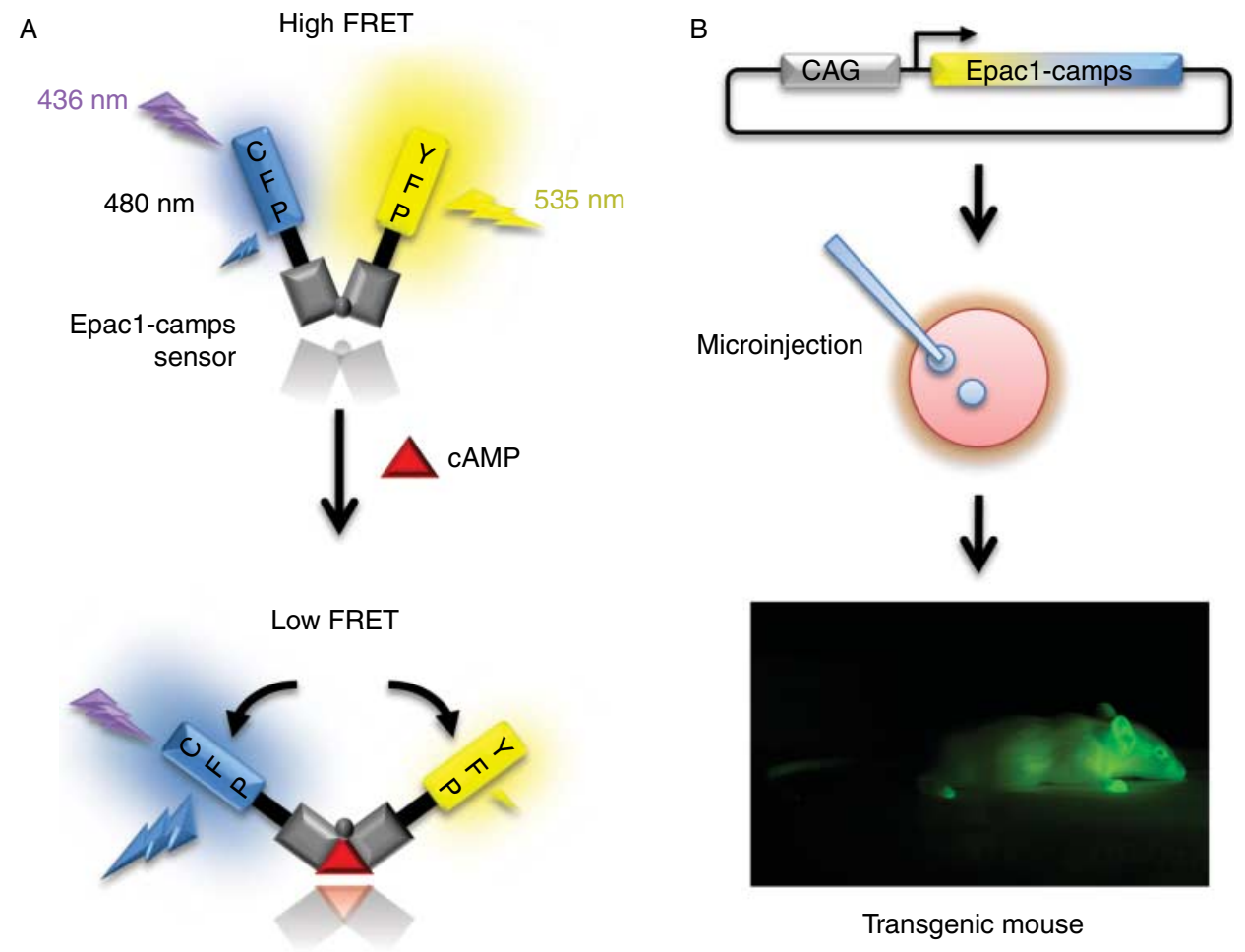

Figure 1 Structure of a single-chain cAMP sensor (Epac1-camps) and generation of a cAMP reporter mouse. (A) The Epac1-camps sensor reports intracellular levels of cAMP based on FRET between CFP and YFP. It consists of CFP and YFP fused to a single CAMP-binding domain derived from the Epac1 protein. In the absence of cAMP, the two fluorophores are in close proximity, and a strong FRET signal is observed. Upon cAMP binding to the sensor, the distance between the two fluorophores increases, leading to a decrease in FRET. (B) Generation of transgenic mice expressing Epac1-camps. To obtain ubiquitous expression, the Epac1-camps sensor was put under the control of a strong constitutive promoter, i.e. the hybrid CMV enhancer/chicken $\beta$-actin (CAG) promoter. Pronuclear injection of this construct gave rise to transgenic mice that express high levels of the sensor in virtually all tissues. Bottom, representative fluorescence image (YFP channel) of a living transgenic mouse showing diffuse strong fluorescence.

activation of adenylyl cyclase and the resulting increase in intracellular cAMP levels (Vassart \& Dumont 1992). As for many other GPCRs, prolonged TSHR stimulation leads to the internalization of both agonist and receptor into endosomes, apparently followed by TSHR recycling to the plasma membrane (Baratti-Elbaz et al. 1999, Lahuna et al. 2005, Frenzel et al. 2006, Calebiro et al. 2009).

Thyroid follicles can be isolated by enzymatic dissociation of thyroid tissue and maintained in culture for over 1 month (Toda et al. 2002, Kusakabe et al. 2006). We have utilized fresh thyroid follicles isolated from Epac1-camps transgenic mice to investigate the spatiotemporal dynamics of TSHR signaling in its native context (Calebiro et al. 2009). A typical setup for performing such experiments consists of an inverted fluorescence microscope equipped with a stable light source and an emission beam splitter (Fig. 3A). Cells are illuminated at the CFP excitation wavelength
(436 nm), and the YFP (FRET) and CFP images are split and simultaneously recorded on a CCD camera. A laminar-flow perfusion system is employed to apply controlled stimuli to the cells. Utilizing this type of setup, we found to our surprise that stimulation of intact thyroid follicles with TSH for $10 \mathrm{~min}$, a time sufficient to induce TSHR internalization into endosomes, is not accompanied by desensitization of the cAMP signal, but rather induces a state of persistent signaling even after removal of the agonist (Fig. 3B). Of note, TSH, internalized together with the TSHR, is found in a perinuclear endocytic compartment containing also $\mathrm{G}_{\mathrm{s}}$ and adenylyl cyclase (Calebiro et al. 2009). The results of these and additional experiments suggest that the TSHR continues to signal to cAMP after internalization and that this intracellular signaling may be required for efficiently triggering specific downstream events, such as the depolymerization of actin cytoskeleton (Calebiro et al. 2009; Fig. 4). 


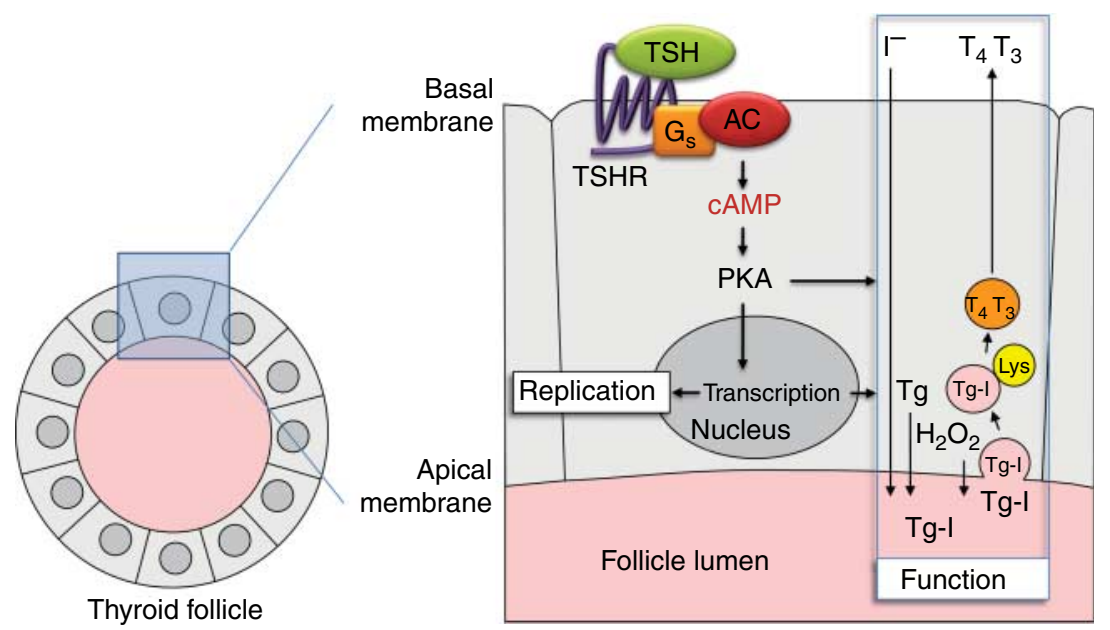

Figure 2 Schematic structure and function of the thyroid follicle. The thyroid follicle constitutes both the anatomical and functional unit of thyroid tissue. It consists of a monolayer of epithelial cells that define an inner cavity, where thyroid hormones are synthesized and stored in the form of iodinated thyroglobulin (Tg-I). Upon Tg-I reuptake from the follicle lumen, thyroid hormones $\left(T_{4}\right.$ and $T_{3}$ ) are released by an enzymatic digestion occurring in lysosomes (Lys). The proteins involved in thyroid hormone secretion and thyroid cell proliferation are under strict transcriptional and post-transcriptional regulation by the TSH/TSHR signaling pathway. TSH binding to the TSHR triggers the $\mathrm{G}_{\mathrm{s}}$-dependent activation of adenylyl cyclase (AC), leading to a stimulation of cAMP production. As a result, PKA is activated and phosphorylates a number of targets that are ultimately responsible for the effects of TSH.

\section{The emerging concept of GPCR signaling at endosomes}

These recent findings on the TSHR provide strong evidence that GPCR signaling to cAMP can continue after internalization. Interestingly, an analogous phenomenon appears to occur also in PTH receptor (PTHR) signaling. The PTHR has two distinct physiological ligands: PTH, a circulating hormone, and PTHrelated peptide (PTHrP), a paracrine factor. These two ligands induce cAMP signals of different duration (Dean et al. 2008). Ferrandon et al. (2009) have recently analyzed PTHR signaling in living cells, utilizing an imaging approach similar to the one we used for the TSHR. Although performed in cells transfected with, and therefore possibly overexpressing, the PTHR, their study suggests that PTH stimulation can induce a rapid internalization of ligand-receptor complexes and adenylyl cyclase into early endosomes. As for the TSHR, internalization of PTHR-PTH complexes is not associated with desensitization of the cAMP response, but rather with persistent signaling. In contrast, PTHrP effects are completely reversible, suggesting that PTHrP may selectively activate receptors at the cell surface.

Furthermore, there is evidence that also $\mathrm{G}_{\mathrm{i}}$-dependent signaling may occur intracellularly. The sphingosine 1-phosphate receptor $1(\mathrm{~S} 1 \mathrm{P} 1)$ is a $\mathrm{G}_{\mathrm{i}} / \mathrm{G}_{\mathrm{q}}$-coupled receptor that serves as a target for the immunomodulatory drug FTY720 (Kappos et al. 2006). A recent study by Müllershausen et al. (2009) indicates that S1P1 receptors may continue signaling to $G_{i}$ after internalization, as shown by a persistent inhibition of forskolin-stimulated cAMP production. In contrast, the $\mathrm{G}_{\mathrm{q}}$-dependent activation of the phospholipase $\mathrm{C} / \mathrm{Ca}^{++}$signaling pathway appears restricted to the cell surface.

The finding that some GPCRs may continue signaling to cAMP after internalization adds to the emerging concept of signaling at endosomes, initially hypothesized and demonstrated for tyrosine kinase receptors (Vieira et al. 1996, Sorkin \& von Zastrow 2009). Indeed, GPCR endocytosis, in addition to playing a role in receptor downregulation, has been shown to have other important functions in regulating and even promoting GPCR signaling. In particular, GPCR endocytosis appears to be required for efficient MAPK signaling by certain GPCRs and might be involved in the $G$ protein-dependent activation of other signaling pathways (Sorkin \& von Zastrow 2009). This is the case for yeast cells, where the GPCR Ste2 has been shown to activate the phosphatidylinositol 3-kinase Vsp34 selectively at endosomes (Slessareva et al. 2006). Similarly, 'non-classical' G protein-dependent signaling pathways at endosomes might also exist in mammalian cells as recently suggested by Diaz Anel (2007) and Garcia-Regalado et al. (2008). 


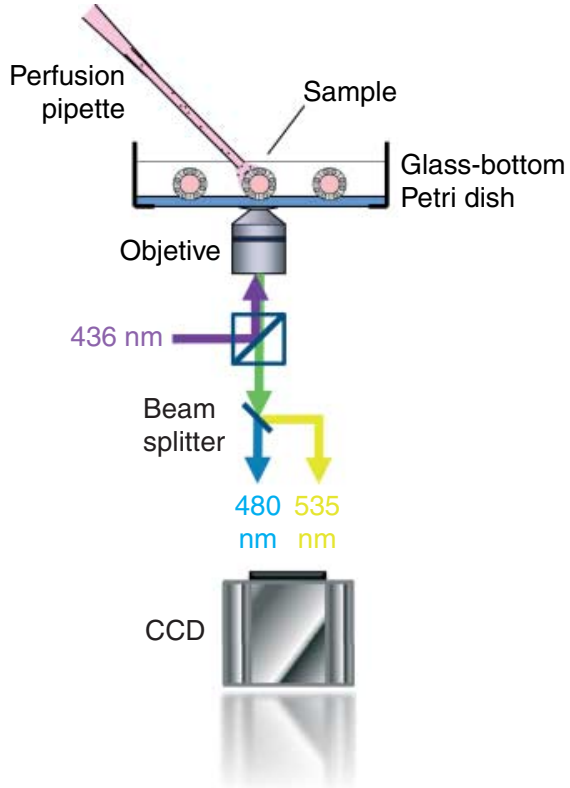

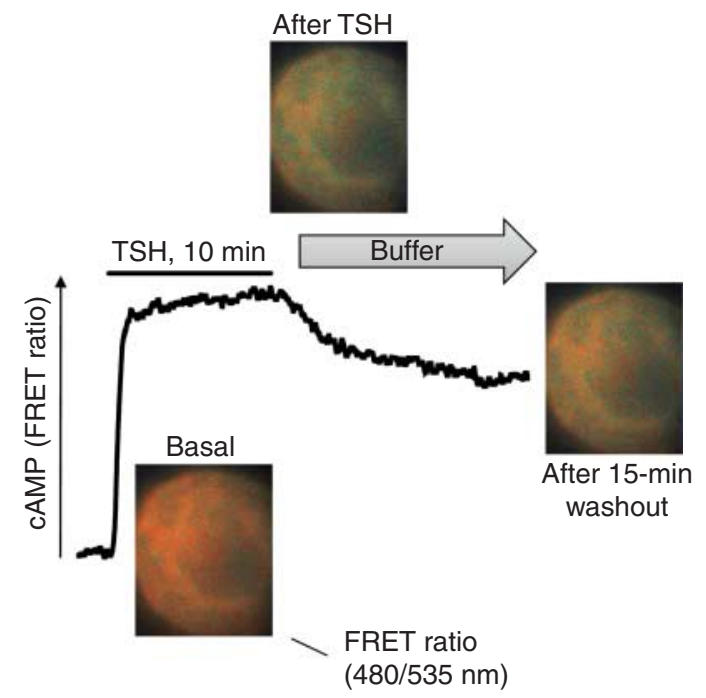

Figure 3 cAMP imaging in living cells by FRET. (A) Typical microscopic setup for real-time FRET imaging, consisting of an inverted fluorescent microscope equipped with a light source for CFP excitation (436 nm). Cells attached to glass-bottom dishes are superfused with various ligands from the tip of a perfusion pipette. CFP $(480 \mathrm{~nm})$ and YFP $(535 \mathrm{~nm})$ fluorescence is split into two channels by a beam splitter device, and the two images (CFP and YFP) are detected using a CCD camera. (B) Representative FRET imaging experiment, in which a thyroid follicle freshly isolated from an Epac1-camps transgenic mouse is stimulated with TSH for $10 \mathrm{~min}$. Hormone application leads to a rapid increase of cAMP levels detected as a change in FRET between CFP and YFP (from red to green in false-color FRET ratio images; note that the FRET ratio is inverted, i.e. calculated as CFP emission/YFP emission, so that FRET ratio values are positively related to intracellular cAMP concentrations). Upon washout of $\mathrm{TSH}$, only an incomplete reversal of the FRET signal is observed, which suggests that the TSHR continues signaling to cAMP after internalization.

The idea that GPCRs may continue to activate G proteins after internalization may appear to contrast with the well-established role of $\beta$-arrestins in GPCR desensitization (Lohse 1993, Pierce et al. 2002). However, although $\beta$-arrestin binding is known to hamper $G$ protein-dependent signaling, some GPCRs, including the TSHR, interact with $\beta$-arrestins only transiently and are internalized without $\beta$-arrestins (Frenzel et al. 2006). In addition, recent studies suggest that different GRKs may produce dissimilar patterns of GPCR phosphorylation and $\beta$-arrestin recruitment, ultimately leading to the selective desensitization of only one of multiple G protein-dependent pathways (Barthet et al. 2009, Zidar et al. 2009). Thus, there seem to be different types of GPCR- $\beta$-arrestin interactions, some of which might be compatible with $\mathrm{G}_{\mathrm{s}}$-dependent signaling. Further studies are required to investigate the role played by GRKs and $\beta$-arrestins in the newly discovered pathway of intracellular GPCR-cAMP signaling.

Another issue to consider is that endosomes may represent only one of several possible intracellular sites of GPCR signaling. Indeed, some GPCRs appear to be localized to a major extent on intracellular membranes, such as those of the endoplasmic reticulum, the Golgi complex, or the nuclear envelope. In addition, there is evidence for early association of GPCRs, G proteins, and GPCR effectors on such intracellular membranes (Dupré et al. 2009). Interestingly, some GPCRs have been reported to signal from the endoplasmic reticulum or the nuclear envelope, either after ligandinduced translocation from the plasma membrane or upon local activation by cell-permeable agonists. Examples of such receptors are the lysophosphatidic acid receptor (Gobeil et al. 2003), GPR30 (Revankar et al. 2005), DFrizzled2 (Mathew et al. 2005), the S1P1 receptor (Liao et al. 2007), and the vasopressin V2 receptor (Robben et al. 2009).

Although the recent findings on the TSHR and PTHR shed new light on the functions of GPCRs at endosomes, we are just beginning to realize their possible implications. A general conclusion one can derive is that cAMP signaling at endosomes tends to be persistent. Of note, several hormones, including TSH (Goichot et al. 1994), and neurotransmitters are secreted in a rhythmic/pulsatile manner and are present at very low concentrations in body fluids. In this respect, sustained cAMP production from internalized receptors may provide a 'memory' mechanism, 


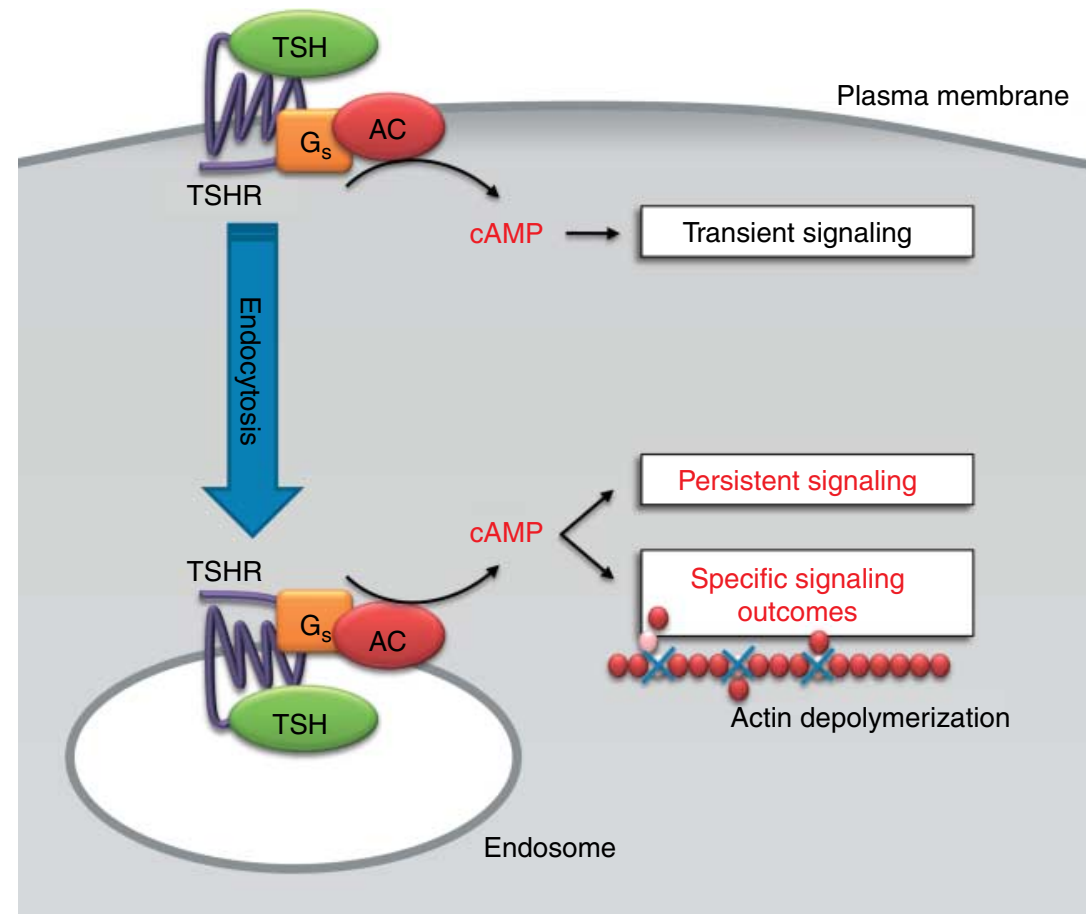

Figure 4 TSHR signaling to cAMP from intracellular compartments. TSH binding to the TSHR activates the cAMP signaling cascade close to the cell surface. Upon prolonged stimulation, both TSH and TSHR are transferred into endosomes. Recent findings suggest that internalized TSH-TSHR complexes continue to stimulate cAMP production at endosomes. This results in persistent signaling and may lead to specific outcomes. For example, persistent receptor signaling from endosomes is apparently involved in the cAMP-mediated effects of TSH on actin depolymerization, an event involved in thyroid hormone release.

allowing cells to prolong their responses after the stimuli have waned (Miaczynska et al. 2004). Furthermore, if this type of intracellular signaling is triggered by certain but not by other agonists, as for the PTHR, it might allow cells to discriminate between different agonists binding to the same receptor.

Moreover, there is initial evidence that cAMP signaling from endosomes might have different outcomes than signaling from the plasma membrane. In the case of the TSHR, signaling from inside the cell is apparently required for triggering full phosphorylation of the vasodilator-stimulated phosphoprotein and actin depolymerization, events that may play a role in thyroglobulin reuptake and, eventually, in thyroid hormone release (Calebiro et al. 2009). To explain these findings, we hypothesize that the TSHR may need to stimulate cAMP production close to an intracellular pool of PKA and/or some downstream targets in order to activate them efficiently. This hypothesis is consistent with the view that, although cAMP is generally thought to diffuse in the cytoplasm, its local concentration may vary considerably between the site of production and more distant sites inside a cell. Such a possibility is supported by the results of mathematical simulations that take into account the localization of adenylyl cyclases on the plasma membrane and most phosphodiesterase activity in the cytosol (Fell 1980). In addition, it is in agreement with some, but not all, experimental findings. Indeed, whether or not cAMP is seen to freely diffuse is apparently dependent on both the cell type and the experimental conditions. Thus, restricted (Bacskai et al. 1993, Hempel et al. 1996) as well as free (Nikolaev et al. 2004) diffusion has been observed in neurons, whereas in cardiac myocytes, both cAMP diffusion (Zaccolo \& Pozzan 2002, Nikolaev et al. 2006) and cAMP-dependent signaling (Jurevicius \& Fischmeister 1996, Warrier et al. 2007) seem to be spatially restrained.

Yet another example of how receptor signaling from the cell interior may differ from that occurring at the cell surface is offered by the case of S1P1 receptors. These receptors are coupled to both $\mathrm{G}_{\mathrm{i}}$ and $\mathrm{G}_{\mathrm{q}}$. Thus, binding of FTY720 phosphate to S1P1 receptors at the cell surface leads to both an inhibition of cAMP production and an increase in intracellular $\mathrm{Ca}^{++}$ concentrations. However, internalized S1P1 receptors seemingly continue to signal to $G_{i}$, thus leading to a persistent reduction of cAMP levels, but stop signaling 
to $\mathrm{G}_{\mathrm{q}}$, with the consequence that $\mathrm{Ca}^{++}$rapidly returns to basal levels (Müllershausen et al. 2009). According to these findings, GPCR internalization may provide a mechanism for a temporal regulation of receptor coupling to different $\mathrm{G}$ protein-dependent pathways.

\section{Concluding remarks}

Based on recent results obtained for the TSHR, PTHR, and S1P1 receptors, we propose a new model of GPCRcAMP signaling. Upon binding of an agonist, GPCRs, which are initially localized at the cell surface, trigger the activation of $G_{s}$ or $G_{i}$, leading to either stimulation or inhibition of cAMP production by adenylyl cyclase. Upon prolonged stimulation, some of these GPCRs are internalized together with their agonists in the endocytic compartment. Here, they may gain access to $\mathrm{G}$ proteins and adenylyl cyclase, which either pre-exist intracellularly or are co-internalized with the receptors. Since both agonists and receptors remain confined in endosomes for some time, this mechanism permits prolonged signaling even after removal of the agonist. In addition, as suggested by the effects of internalized TSHR on actin cytoskeleton, this new type of signaling may produce specific functional outcomes.

These findings were mostly obtained by monitoring cAMP signaling directly in living cells and, in the case of the TSHR, in the multicellular functional unit of thyroid tissue, the thyroid follicle. Thus, they represent a significant step ahead in the direction of observing the spatiotemporal dynamics of GPCR signaling in its natural context. These experiments were largely made possible by the recent development of fluorescent reporters for the GPCR-cAMP signaling pathway and by the successful generation of a transgenic mouse model expressing one of these sensors. In our opinion, similar types of imaging approaches, hopefully taking advantage of improved sensor design and microscopy techniques, will play a major role in further investigations aimed at unraveling the complex spatiotemporal dynamics of GPCR signaling cascades.

While a new scenario for the life of GPCRs after internalization is beginning to emerge, there is a series of important issues that need to be addressed. These include questions such as: is intracellular signaling to cAMP a general feature of $\mathrm{G}_{\mathrm{s}} / \mathrm{G}_{\mathrm{i}}$-coupled receptors or is it a peculiar characteristic of only certain receptors? May GPCRs activate also other G proteins at intracellular sites? What is the physiological and pathophysiological impact of GPCR signaling at endosomes? Is this phenomenon of relevance for drug design and can it be exploited for pharmacological purposes? Although further studies will be required to answer these and other important questions, endosomes should no longer be considered as passive carriers for GPCRs en route to degradation, but rather as specialized signaling platforms capable of affecting the specificity as well as spatiotemporal dynamics of GPCR signaling.

\section{Declaration of interest}

The authors declare that there is no conflict of interest that could be perceived as prejudicing the impartiality of the research reported.

\section{Funding}

Research by the authors referred to in this publication is supported by grants from the European Research Council (Advanced Grant TOPAS) and the Deutsche Forschungsgemeinschaft (SFB487).

\section{References}

Adams SR, Harootunian AT, Buechler YJ, Taylor SS \& Tsien RY 1991 Fluorescence ratio imaging of cyclic AMP in single cells. Nature 349 694-697.

Bacskai BJ, Hochner B, Mahaut-Smith M, Adams SR, Kaang BK, Kandel ER \& Tsien RY 1993 Spatially resolved dynamics of cAMP and protein kinase A subunits in Aplysia sensory neurons. Science $260222-226$.

Baratti-Elbaz C, Ghinea N, Lahuna O, Loosfelt H, Pichon C \& Milgrom E 1999 Internalization and recycling pathways of the thyrotropin receptor. Molecular Endocrinology 13 1751-1765.

Barthet G, Carrat G, Cassier E, Barker B, Gaven F, Pillot M, Framery B, Pellissier LP, Augier J, Kang DS et al. 2009 ß-Arrestin1 phosphorylation by GRK5 regulates $\mathrm{G}$ protein-independent $5-\mathrm{HT}_{4}$ receptor signalling. EMBO Journal 28 2706-2718.

Bünemann M, Frank M \& Lohse MJ $2003 \mathrm{G}_{\mathrm{i}}$ protein activation in intact cells involves subunit rearrangement rather than dissociation. PNAS 100 16077-16082.

Calebiro D, Nikolaev VO, Gagliani MC, de Filippis T, Dees C, Tacchetti C, Persani L \& Lohse MJ 2009 Persistent cAMP-signals triggered by internalized G-protein-coupled receptors. PLoS Biology 7 e1000172.

Castro M, Nikolaev VO, Palm D, Lohse MJ \& Vilardaga JP 2005 Turnon switch in parathyroid hormone receptor by a two-step parathyroid hormone binding mechanism. PNAS 102 16084-16089.

Dean T, Vilardaga JP, Potts JT Jr \& Gardella TJ 2008 Altered selectivity of parathyroid hormone (PTH) and PTH-related protein (PTHrP) for distinct conformations of the $\mathrm{PTH} / \mathrm{PTH} \mathrm{P}$ receptor. Molecular Endocrinology 22 156-166.

Diaz Anel AM 2007 Phospholipase C $\beta 3$ is a key component in the $\mathrm{G} \beta \gamma / \mathrm{PKC \eta} / \mathrm{PKD}$-mediated regulation of trans-Golgi network to plasma membrane transport. Biochemical Journal 406 157-165.

DiPilato LM, Cheng X \& Zhang J 2004 Fluorescent indicators of cAMP and Epac activation reveal differential dynamics of cAMP signaling within discrete subcellular compartments. PNAS 101 16513-16518.

Dupré DJ, Robitaille M, Rebois RV \& Hebert TE 2009 The role of G $\beta \gamma$ subunits in the organization, assembly, and function of GPCR signaling complexes. Annual Review of Pharmacology and Toxicology $4931-56$.

Fell DA 1980 Theoretical analyses of the functioning of the high- and low- $K_{\mathrm{m}}$ cyclic nucleotide phosphodiesterases in the regulation of the concentration of adenosine $3^{\prime}, 5^{\prime}$-cyclic monophosphate in animal cells. Journal of Theoretical Biology 84 361-385.

Ferrandon S, Feinstein TN, Castro M, Wang B, Bouley R, Potts JT, Gardella TJ \& Vilardaga JP 2009 Sustained cyclic AMP production by parathyroid hormone receptor endocytosis. Nature Chemical Biology 5 734-742.

Frenzel R, Voigt C \& Paschke R 2006 The human thyrotropin receptor is predominantly internalized by $\beta$-arrestin 2. Endocrinology 147 3114-3122. 
Garcia-Regalado A, Guzman-Hernandez ML, Ramirez-Rangel I, Robles-Molina E, Balla T, Vazquez-Prado J \& Reyes-Cruz G 2008 G protein-coupled receptor-promoted trafficking of $G \beta 1 \gamma 2$ leads to AKT activation at endosomes via a mechanism mediated by

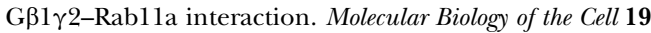
4188-4200.

Gilman AG 1987 G proteins: transducers of receptor-generated signals. Annual Review of Biochemistry 56 615-649.

Gobeil F Jr, Bernier SG, Vazquez-Tello A, Brault S, Beauchamp MH, Quiniou C, Marrache AM, Checchin D, Sennlaub F, Hou X et al. 2003 Modulation of pro-inflammatory gene expression by nuclear lysophosphatidic acid receptor type-1. Journal of Biological Chemistry 278 38875-38883.

Goichot B, Brandenberger G, Saini J, Wittersheim G \& Follenius M 1994 Nycthemeral patterns of thyroid hormones and their relationships with thyrotropin variations and sleep structure. Journal of Endocrinological Investigation 17 181-187.

Hanyaloglu AC \& von Zastrow M 2008 Regulation of GPCRs by endocytic membrane trafficking and its potential implications. Annual Review of Pharmacology and Toxicology 48 537-568.

Hempel CM, Vincent P, Adams SR, Tsien RY \& Selverston AI 1996 Spatio-temporal dynamics of cyclic AMP signals in an intact neural circuit. Nature 384 166-169.

Janetopoulos C, Jin T \& Devreotes P 2001 Receptor-mediated activation of heterotrimeric G-proteins in living cells. Science 291 2408-2411.

Jurevicius J \& Fischmeister R 1996 cAMP compartmentation is responsible for a local activation of cardiac $\mathrm{Ca}^{2+}$ channels by $\beta$-adrenergic agonists. PNAS 93 295-299.

Kappos L, Antel J, Comi G, Montalban X, O'Connor P, Polman CH, Haas T, Korn AA, Karlsson G \& Radue EW 2006 Oral fingolimod (FTY720) for relapsing multiple sclerosis. New England Journal of Medicine 355 1124-1140.

Krueger KM, Daaka Y, Pitcher JA \& Lefkowitz RJ 1997 The role of sequestration in $\mathrm{G}$ protein-coupled receptor resensitization. Regulation of $\beta_{2}$-adrenergic receptor dephosphorylation by vesicular acidification. Journal of Biological Chemistry 272 5-8.

Kusakabe T, Kawaguchi A, Hoshi N, Kawaguchi R, Hoshi S \& Kimura S 2006 Thyroid-specific enhancer-binding protein/NKX2.1 is required for the maintenance of ordered architecture and function of the differentiated thyroid. Molecular Endocrinology 20 1796-1809.

Lagerstrom MC \& Schioth HB 2008 Structural diversity of G proteincoupled receptors and significance for drug discovery. Nature Reviews. Drug Discovery 7 339-357.

Lahuna O, Quellari M, Achard C, Nola S, Meduri G, Navarro C, Vitale N, Borg JP \& Misrahi M 2005 Thyrotropin receptor trafficking relies on the hScrib-BPIX-GIT1-ARF6 pathway. EMBO Journal 24 1364-1374.

Liao JJ, Huang MC, Graler M, Huang Y, Qiu H \& Goetzl EJ 2007 Distinctive T cell-suppressive signals from nuclearized type 1 sphingosine 1-phosphate $\mathrm{G}$ protein-coupled receptors. Journal of Biological Chemistry 282 1964-1972.

Lohse MJ 1993 Molecular mechanisms of membrane receptor desensitization. Biochimica et Biophysica Acta 1179 171-188.

Lohse MJ, Nikolaev VO, Hein P, Hoffmann C, Vilardaga JP \& Bünemann M 2008 Optical techniques to analyze real-time activation and signaling of G-protein-coupled receptors. Trends in Pharmacological Sciences 29 159-165.

Magner JA 1990 Thyroid-stimulating hormone: biosynthesis, cell biology, and bioactivity. Endocrine Reviews 11 354-385.

Mathew D, Ataman B, Chen J, Zhang Y, Cumberledge S \& Budnik V 2005 Wingless signaling at synapses is through cleavage and nuclear import of receptor DFrizzled2. Science 310 1344-1347.

Miaczynska M, Pelkmans L \& Zerial M 2004 Not just a sink: endosomes in control of signal transduction. Current Opinion in Cell Biology 16 400-406.
Müllershausen F, Zecri F, Cetin C, Billich A, Guerini D \& Seuwen K 2009 Persistent signaling induced by FTY720-phosphate is mediated by internalized S1P1 receptors. Nature Chemical Biology 5 428-434.

Nikolaev VO, Bünemann M, Hein L, Hannawacker A \& Lohse MJ 2004 Novel single chain cAMP sensors for receptor-induced signal propagation. Journal of Biological Chemistry 279 37215-37218.

Nikolaev VO, Bünemann M, Schmitteckert E, Lohse MJ \& Engelhardt S 2006 Cyclic AMP imaging in adult cardiac myocytes reveals farreaching $\beta_{1}$-adrenergic but locally confined $\beta_{2}$-adrenergic receptormediated signaling. Circulation Research 99 1084-1091.

Pierce KL, Premont RT \& Lefkowitz RJ 2002 Seven-transmembrane receptors. Nature Reviews. Molecular Cell Biology 3 639-650.

Pippig S, Andexinger S \& Lohse MJ 1995 Sequestration and recycling of $\beta_{2}$-adrenergic receptors permit receptor resensitization. Molecular Pharmacology 47 666-676.

Ponsioen B, Zhao J, Riedl J, Zwartkruis F, van der Krogt G, Zaccolo M, Moolenaar WH, Bos JL \& Jalink K 2004 Detecting cAMP-induced Epac activation by fluorescence resonance energy transfer: Epac as a novel cAMP indicator. EMBO Reports 5 1176-1180.

Revankar CM, Cimino DF, Sklar LA, Arterburn JB \& Prossnitz ER 2005 A transmembrane intracellular estrogen receptor mediates rapid cell signaling. Science 307 1625-1630.

Robben JH, Kortenoeven ML, Sze M, Yae C, Milligan G, Oorschot VM, Klumperman J, Knoers NV \& Deen PM 2009 Intracellular activation of vasopressin V2 receptor mutants in nephrogenic diabetes insipidus by nonpeptide agonists. PNAS 106 12195-12200.

Slessareva JE, Routt SM, Temple B, Bankaitis VA \& Dohlman HG 2006 Activation of the phosphatidylinositol 3-kinase $\mathrm{Vps} 34$ by a $\mathrm{G}$ protein $\alpha$ subunit at the endosome. Cell 126 191-203.

Sorkin A \& von Zastrow M 2009 Endocytosis and signalling: intertwining molecular networks. Nature Reviews. Molecular Cell Biology 10 609-622.

Toda S, Watanabe K, Yokoi F, Matsumura S, Suzuki K, Ootani A, Aoki S, Koike N \& Sugihara H 2002 A new organotypic culture of thyroid tissue maintains three-dimensional follicles with $\mathrm{C}$ cells for a long term. Biochemical and Biophysical Research Communications 294 906-911.

Tsao P, Cao T \& von Zastrow M 2001 Role of endocytosis in mediating downregulation of G-protein-coupled receptors. Trends in Pharmacological Sciences 22 91-96.

Vassart G \& Dumont JE 1992 The thyrotropin receptor and the regulation of thyrocyte function and growth. Endocrine Reviews 13 596-611.

Vieira AV, Lamaze C \& Schmid SL 1996 Control of EGF receptor signaling by clathrin-mediated endocytosis. Science 274 2086-2089.

Vilardaga JP, Bünemann M, Krasel C, Castro M \& Lohse MJ 2003 Measurement of the millisecond activation switch of $G$ proteincoupled receptors in living cells. Nature Biotechnology 21 807-812.

Warrier S, Ramamurthy G, Eckert RL, Nikolaev VO, Lohse MJ \& Harvey RD 2007 cAMP microdomains and L-type $\mathrm{Ca}^{2+}$ channel regulation in guinea-pig ventricular myocytes. Journal of Physiology $\mathbf{5 8 0} 765-776$.

Yu SS, Lefkowitz RJ \& Hausdorff WP 1993 $\beta$-Adrenergic receptor sequestration. A potential mechanism of receptor resensitization. Journal of Biological Chemistry 268 337-341.

Zaccolo M \& Pozzan T 2002 Discrete microdomains with high concentration of cAMP in stimulated rat neonatal cardiac myocytes. Science 295 1711-1715.

Zaccolo M, De Giorgi F, Cho CY, Feng L, Knapp T, Negulescu PA, Taylor SS, Tsien RY \& Pozzan T 2000 A genetically encoded, fluorescent indicator for cyclic AMP in living cells. Nature Cell Biology 2 25-29.

Zidar DA, Violin JD, Whalen EJ \& Lefkowitz RJ 2009 Selective engagement of $\mathrm{G}$ protein coupled receptor kinases (GRKs) encodes distinct functions of biased ligands. PNAS 106 9649-9654.

Received in final form 20 March 2010

Accepted 8 April 2010

Made available online as an Accepted Preprint 8 April 2010 